\title{
Thermal motion of carbon clusters and production of carbon nanotubes by gravity-free arc discharge
}

\author{
T. Mieno ${ }^{\mathrm{a})}$ \\ Department of Physics, Shizuoka University, Ooya, Suruga-ku, Shizuoka 422-8529, Japan \\ M. Takeguchi \\ National Institute for Material Science, Sakura, Tsukuba 305-0003, Japan
}

(Received 19 July 2005; accepted 16 March 2006; published online 23 May 2006)

\begin{abstract}
Thermal and diffusion properties of hot gas around a dc arc discharge under a gravity-free condition are investigated using a jet plane in order to improve the arc production of carbon clusters. Spherically symmetric temperature distribution of $\mathrm{He}$ gas around the arc plasma and monotonic slow expansion of the high-temperature region are observed. By means of the passive-type Mie scattering method, random slow diffusion of carbon clusters around the arc plasma is clearly observed under the gravity-free condition. This indicates that carbon clusters including single-walled carbon nanotubes are synthesized around the arc plasma where the He temperature is higher than $1000 \mathrm{~K}$. It is confirmed that large bundles of fatter single-walled carbon nanotubes are produced under the gravity-free condition. (C) 2006 American Institute of Physics.
\end{abstract}

[DOI: $10.1063 / 1.2194122]$

\section{INTRODUCTION}

At the end of the 20th century, a mass-production method of stable carbon clusters, such as fullerenes and nanotubes, was developed, ${ }^{1-4}$ and potential applications of these clusters have been actively investigated. Although there are many production methods, they do not have sufficient efficiency, and selective production of carbon nanotubes (CNTs) with the desired diameter, length, and chirality has not been achieved. More efficient production methods should be developed. The carbon clusters are produced by the arc-discharge method in subatmospheric He gas, where strong heat convection causes sublimated carbon atoms to immediately flow upwards, and the reaction time in hot gas is restricted. The cooling and cohesion process of carbon clusters is important for the synthesis of fullerenes and carbon nanotubes.

In order to examine the effects of gravity and heat convection on arc production, a 12-m-high vertical-swing tower was constructed and endohedral metallofullerenes and single-walled carbon nanotubes ${ }^{5,6}$ (SWNTs) were produced under repetitive gravity-free conditions. These experiments revealed a dramatic increase of production efficiencies of metallofullerenes and SWNTs.

In order to clarify the gravity-free effect, ${ }^{7,8}$ the gas temperature distribution under the gravity-free condition is investigated using the parabolic flight of a jet plane, and the result is compared with that obtained under the normalgravity condition. ${ }^{9}$ The production location and diffusion characteristics of carbon clusters around the arc are measured by the Mie scattering method. Properties of SWNTs produced under the two gravity conditions are compared.

Preliminary results have been presented in a letter and at a conference., ${ }^{9,10}$

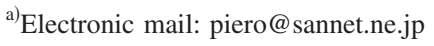

\section{THEORY}

When carbon clusters of the same diameter and mass are produced in a globe with radius $r_{0}$ located at the center of spherical coordinates and the clusters diffuse isotropically with diffusion coefficient $D_{\mathrm{C}}$ without any chemical reaction in a spherical vessel with wall radius of $r_{1}\left(r_{1}>r_{0}\right)$, which is located concentrically around the globe, we can calculate the density profile of the carbon clusters $n_{\mathrm{C}}(r)$ and radial particle flux of carbon clusters $J_{\mathrm{C}}(r)$ from the Fick's diffusion laws. ${ }^{11}$ Under the steady-state condition,

$$
\begin{aligned}
& n_{\mathrm{C}}(r)=\frac{r_{1} r_{0}}{r_{1}-r_{0}}\left(n_{0}-n_{1}\right) \frac{1}{r}+\frac{r_{1} n_{1}-r_{0} n_{0}}{r_{1}-r_{0}}, \\
& J_{\mathrm{C}}(r)=D_{\mathrm{C}} \frac{r_{1} r_{0}}{r_{1}-r_{0}}\left(n_{0}-n_{1}\right) \frac{1}{r^{2}},
\end{aligned}
$$

where $n_{0}$ and $n_{1}$ are the cluster densities at $r=r_{0}$ and $r=r_{1}$, respectively. When the carbon clusters diffuse in $\mathrm{He}$ gas, according to the classical diffusion theory, ${ }^{12}$ their diffusion coefficient $D_{\mathrm{CHe}}$ is written as

$$
D_{\mathrm{CHe}}=\frac{4}{3} \frac{\sqrt{2}}{\pi^{3 / 2}}\left(\frac{1}{m_{\mathrm{C}}}+\frac{1}{m_{\mathrm{He}}}\right)^{1 / 2} \frac{\left(\kappa_{B} T\right)^{3 / 2}}{p\left(d_{\mathrm{C}}+d_{\mathrm{He}}\right)^{2}},
$$

where $m_{\mathrm{C}}$ and $m_{\mathrm{He}}$ are the cluster mass and He-atom mass, respectively. $\kappa_{\mathrm{B}}, T, p, d_{\mathrm{C}}$, and $d_{\mathrm{He}}$ are Boltzmann's constant, gas temperature, gas pressure, diameter of the cluster, and diameter of the He atom, respectively. From this equation, the radial diffusion velocity of carbon clusters can be estimated. Figure 1 shows the calculated results of diffusion velocities of $\mathrm{C}_{2}$ and $\mathrm{C}_{60}$ molecules versus $\mathrm{He}$ pressure, where the calculated position $r$ is $3 \mathrm{~cm}$ from the arc center, $T_{\mathrm{He}}$ $=5000 \mathrm{~K}$, and the vessel size is the same as that of the experimental chamber. The natural convection velocity of $\mathrm{He}$, by which all the carbon clusters are brought up, is calculated using a commercially available simulation program (Mizuho 


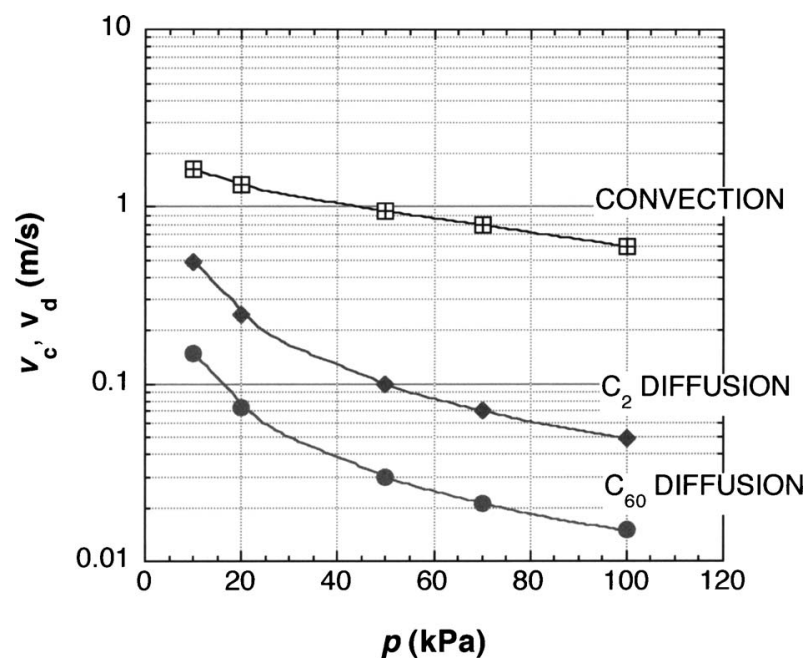

FIG. 1. (Color online) He pressure dependence of thermal diffusion velocities $V_{d}$ of $\mathrm{C}_{2}$ and $\mathrm{C}_{60}$ molecules and heat-convection velocity $V_{\mathrm{C}}$ of $\mathrm{He}$ gas under the normal-gravity condition. Calculation for $T=5000 \mathrm{~K}$ and $r$ $=3.0 \mathrm{~cm}$.

Information \& Research Institute, Inc., Fuji-RIC/Alfa-Flow) with a UNIX workstation. In the simulation, a globe-type heat source with a radius of $0.50 \mathrm{~cm}$ is set at the center of cylindrical coordinates, and the time evolution of the spatial distribution of gas temperature and gas velocity on an $r-z$ coordinate plane under the normal-gravity condition is calculated. The main calculation method is the simplified marker and cell (SMAC) method. ${ }^{13,14}$ The calculated heatconvection velocity under normal gravity is also shown in Fig. 1. From the results of these calculations, it can be predicted that heavier and larger carbon clusters diffuse much more slowly under the gravity-free condition. As the gas temperature monotonically decreases at the edge of the plasma flame, large carbon clusters cool much more slowly under the gravity-free condition. ${ }^{15}$

From the fluid simulation, the time evolution of temperature contours of He gas around the arc flame can be calculated. Figure 2(a) shows the temperature contour (side view) under the gravity-free condition at $t=1.0 \mathrm{~s}$ after the arc starts, where $p=40 \mathrm{kPa}$ and the region of $T=500-5000 \mathrm{~K}$ is shown. The frame in the graph shows the vertical axis and the boundary wall. Under this gravity condition, the hot-gas region monotonically and isotropically expands, and it takes a long time to reach a steady-state condition. Meanwhile, under the normal-gravity condition, temperature contours are calculated as shown in Fig. 2(b), where $t=0.60 \mathrm{~s}$ and $p$ $=40 \mathrm{kPa}$ and the region of $T=500-5000 \mathrm{~K}$ is shown. Under this gravity condition, the temperature contours attain a steady state within $0.5 \mathrm{~s}$, and thereafter, there is almost no change. The particles and the thermal energy are continuously brought to the upper end boundary. At $t=0.50 \mathrm{~s}$ after the arc is turned on, approximate volumes of the hightemperature region $(T=5000-2000 \mathrm{~K})$ under acceleration of gravity $G=0 g_{0}$ and $G=1 g_{0}$ ( $g_{0}$ : normal acceleration of gravity) are calculated and the results are shown in Fig. 2(c). The difference is clear and the volume is about five times larger under $G=0 g_{0}$.

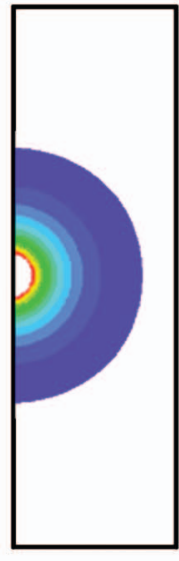

(a)

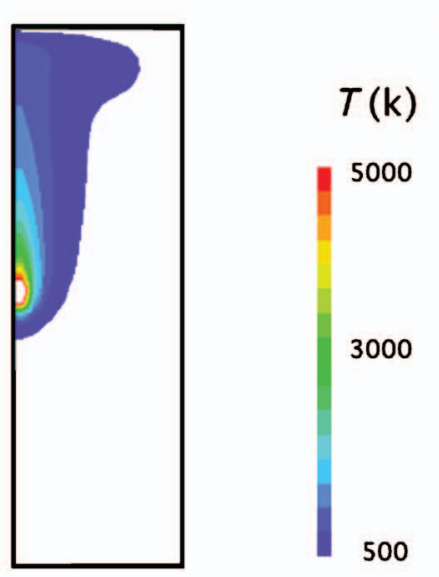

(b)

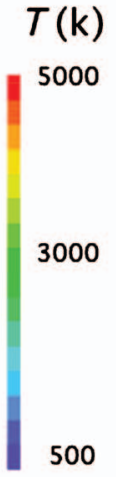

(k)

5000

000

(c)

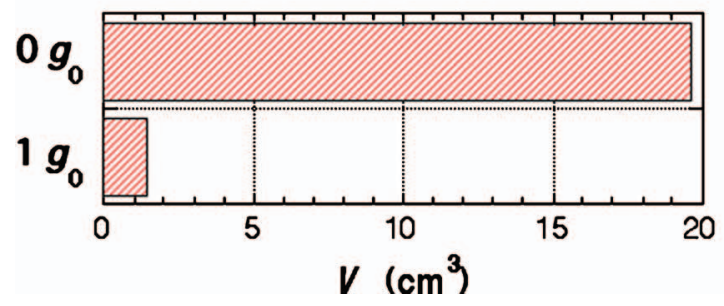

FIG. 2. (Color) Simulation results of temperature contours $(T$ $=500-5000 \mathrm{~K}$ ) for the two gravity conditions: (a) $G=0, t=1.0 \mathrm{~s}, p(\mathrm{He})$ $=40 \mathrm{kPa}$ and (b) $G=1 g_{0}, t=0.60 \mathrm{~s}, p(\mathrm{He})=40 \mathrm{kPa}$. (c) Volumes of hightemperature region $(T=5000-2000 \mathrm{~K})$ calculated under the two gravity conditions at $0.50 \mathrm{~s}$ after the arc is turned on. The frames of (a) and (b) show the vertical axis and the boundary wall.

\section{EXPERIMENTAL SETUP AND METHOD}

The entire experimental setup is installed on two aluminum racks specially prepared for a jet plane (Grumman G-II operated by Diamond Air Service, Japan); the schematic is shown in Fig. 3. A metal arc reactor, a pumping system, a gas-feeding system, a dc power supply, a video camera, an array of thermocouples, and a recording system are installed. In the cylindrical arc reactor of $11.5 \mathrm{~cm} \phi$ and $19.4 \mathrm{~cm}$ height, two carbon electrodes (a $6.0 \mathrm{~mm} \phi$ cylindrical anode and a $10 \mathrm{~mm} \phi$ cylindrical cathode) are set. After evacuation, He gas is introduced and the reactor is closed.

The jet plane takes off from Nagoya airport and the experiment is performed over the Pacific Ocean at a height of 


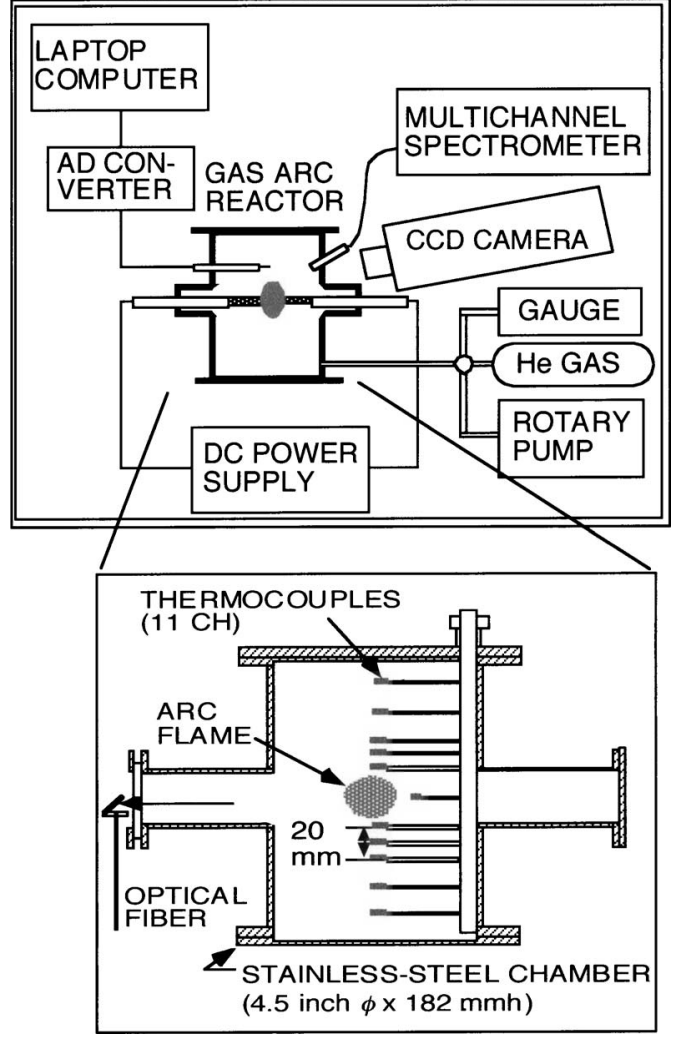

FIG. 3. (Color online) Schematic of experimental setup in a jet plane.

$21000-28000 \mathrm{ft}$. In one flight, the experiment is limited to $1 \mathrm{~h}$. Ten to twenty parabolic flights are carried out at certain time intervals, the machine being recovered and the next experimental conditions being set each time. After the jet plane goes into parabolic flight and the gravity becomes zero, the arc is turned on by an operator and maintained for about $40 \mathrm{~s}$ ( $20 \mathrm{~s}$ of gravity-free time, $G=0$, and $20 \mathrm{~s}$ of $G$ $\left.=1.5 g_{0}\right)$.

The time dependence of the vertical distribution of gas temperature over and under the arc is measured with 11 thermocouples. The analog data are digitized using a special analog-to-digital (AD) converter and stored in a laptop computer. Under the gravity-free condition, the gas temperature distribution is symmetric and it expands radially and monotonically. During the $20 \mathrm{~s}$ of gravity-free time, which is the maximum gravity-free time in this experiment, the temperature profile cannot be stabilized. A longer gravity-free time appears to be necessary to attain the steady state, with a high-temperature and larger volume sphere.

In order to clarify the production location of carbon clusters and their diffusion speed, the passive Mie scattering method is adopted. By removing the array of thermocouples and using a metal-obstacle plate, the direct light from the arc flame is eliminated and only light scattered by the produced carbon clusters is recorded by a video camera, whereby a vertical profile of the produced carbon clusters and their diffusion can be observed.

In this parabolic flight experiment, SWNTs are produced under the gravity-free condition. These SWNTs are collected from the reactor and observed using a transmission electron

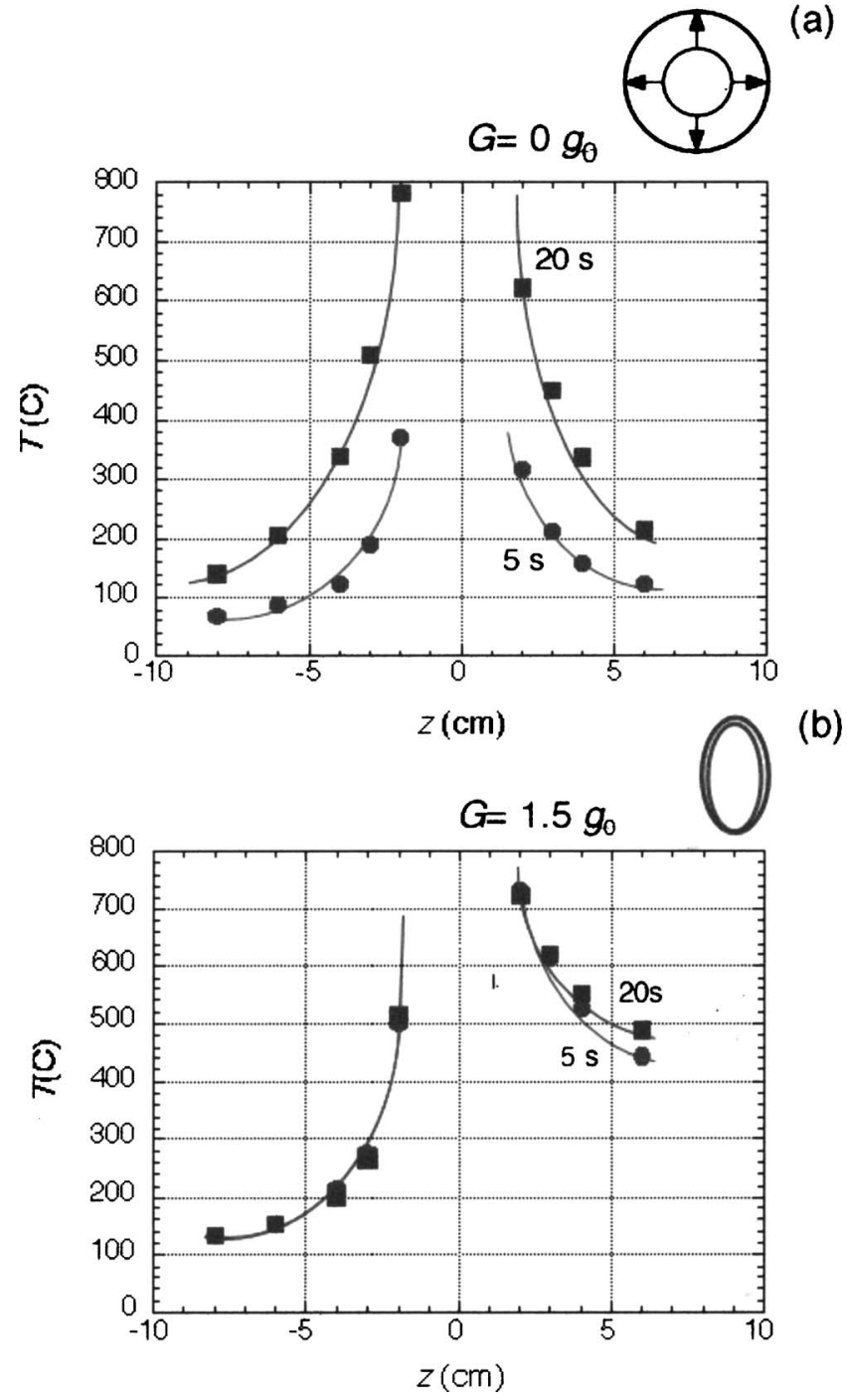

FIG. 4. (Color online) Vertical temperature distributions over and under the arc at $\Delta t=5.0$ and $20 \mathrm{~s}$ (a) after gravity becomes 0 and (b) after gravity becomes $1.5 g_{0} \cdot p(\mathrm{He})=70 \mathrm{kPa}$ and $I_{d}=40 \mathrm{~A}$.

microscope (TEM). The diameter distribution, shapes of bundles, and impurity content of the samples are measured.

\section{EXPERIMENTAL RESULTS AND DISCUSSION}

Figure 4 shows gas temperature distributions along the vertical axis under the two gravities $G=0 g_{0}$ and $1.5 g_{0}$, where $\mathrm{He}$ pressure $p=70 \mathrm{kPa}$ and discharge current $I_{d}$ $=40 \mathrm{~A}$. When the gravity becomes 0 , the arc is turned on and the hot-gas region monotonically and symmetrically expands with arc time $\Delta t$. It is conjectured that the heat flow is suppressed under the convection-free condition, and the generated Ohmic heat tends to be concentrated into a spherical large-volume hot-gas region. The model of the temperature contours is roughly illustrated at the top of Fig. 4(a) and is consistent with the simulation result. It is confirmed that a steady-state, spherical hot-gas region of large volume can be generated when a gravity-free time longer than $20 \mathrm{~s}$ is realized. From the data in Fig. 4(a), the expansion speed of the $T=500 \mathrm{~K}$ temperature contour can be calculated; it is about $8 \mathrm{~cm} / \mathrm{min}$, which is very slow. This expansion does not stop 


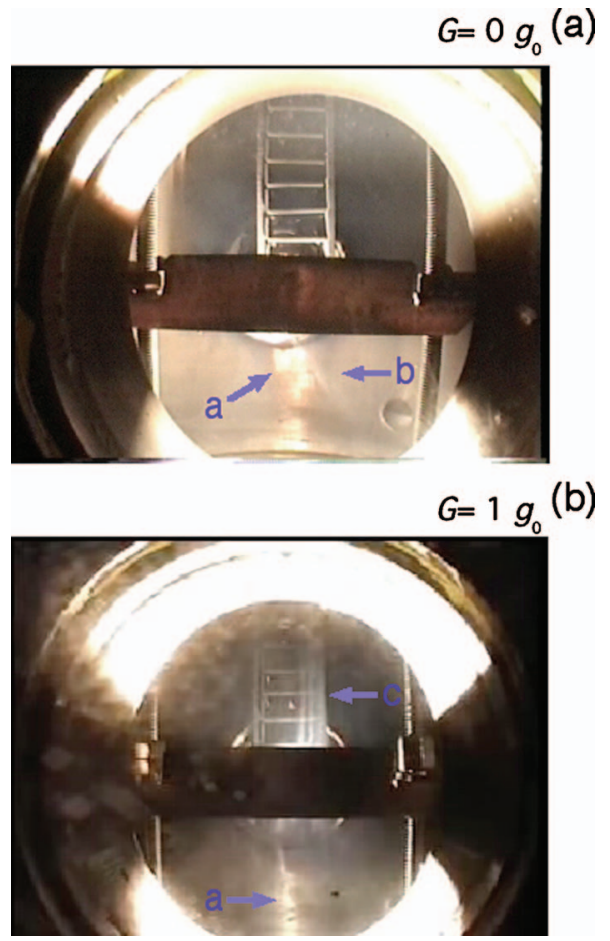

FIG. 5. (Color) Mie scattered light observed from a side port under (a) $G$ $=0$ and (b) $G=1 g_{0}$. SWNTs are synthesized around the arc flame. $p(\mathrm{He})$ $=40 \mathrm{kPa}, I_{d} \sim 50 \mathrm{~A}$.

within $20 \mathrm{~s}$. Under the normal-gravity condition, the gas temperature distribution is as shown in Fig. 4(b). Under this condition, steady-state convection flow is immediately formed and the thermal energy continuously diffuses to the upper region. Therefore, the hot-gas region is not symmetrical and has a smaller volume, which is also consistent with the simulation result.

By using an obstacle plate, the passive Mie scattering from the carbon clusters can be measured. In this case, a $6.0 \times 6.0 \mathrm{~mm}^{2}$ rectangular metal-mixed carbon $\operatorname{rod}(\mathrm{Ni}$ of $4.2 \mathrm{~mol} \%$, Y of $0.90 \mathrm{~mol} \%$ included in carbon) is used as a sublimation source to make SWNTs, where $p(\mathrm{He})=40 \mathrm{kPa}$ and $I_{d} \sim 50 \mathrm{~A}$. The typical images for the two gravity conditions are shown in Fig. 5. Under the gravity-free condition, the density distribution of the carbon clusters in the reactor is almost spherically symmetric. The clusters move around randomly and their expansion speed is less than $1 \mathrm{~cm} / \mathrm{s}$. The emissions indicated by "Arrow a" in Figs. 5(a) and 5(b) are reflections from the inner wall. In the photographs, strong reflection from the lower sidewall would confuse the situation of homogeneous diffusion. There is a thin spout from the anode, indicated by "Arrow b," which is sometimes recognized in the image. It is conjectured that this spout is caused by an inhomogeneous electron flow to the anode and disturbs the slow cluster reaction. Technical improvement is necessary to remove this kind of spout. On the other hand, when the gravity is $1 g_{0}$, the behavior is completely different. Under this gravity, the clusters appear only above the arc plasma, and they flow monotonically upward at a speed of about $0.5 \mathrm{~m} / \mathrm{s}$, which is maintained by natural heat convection. The constant flow of carbon clusters is indicated by "Arrow c" in Fig. 5(b). From the results of these measure-

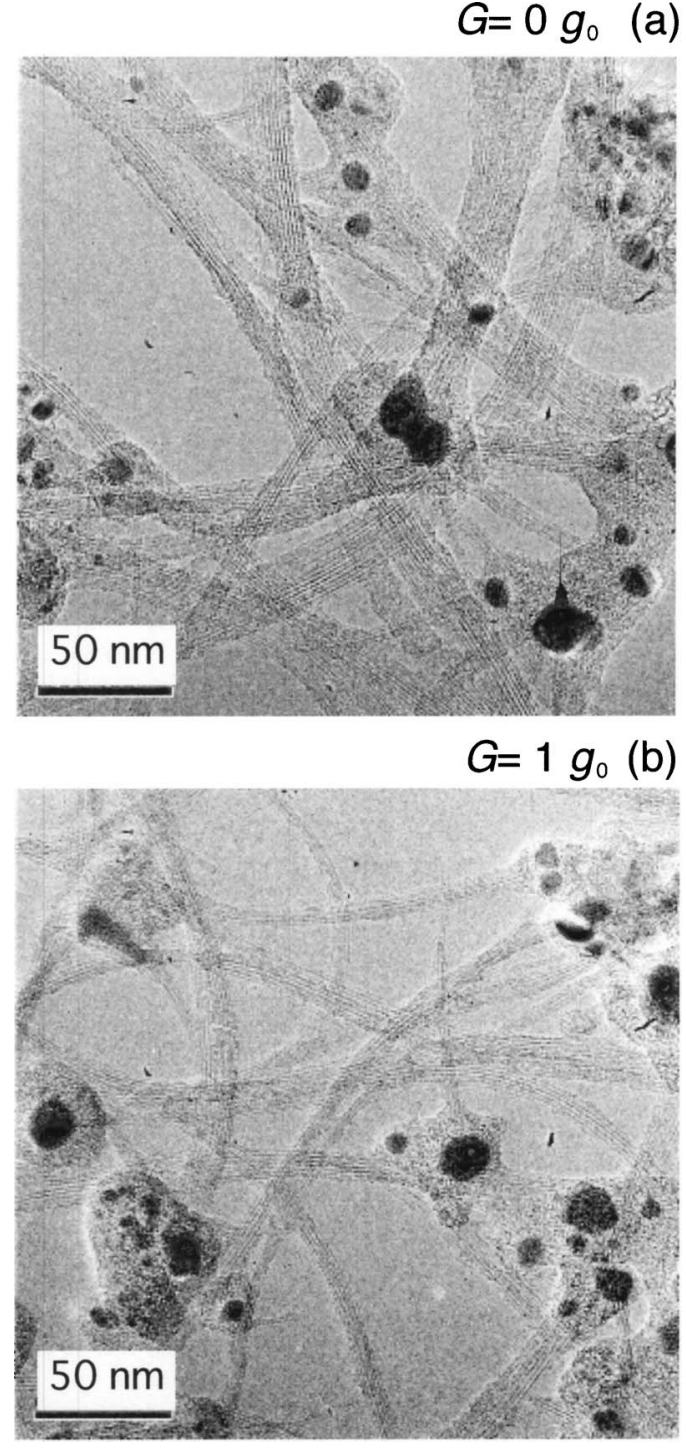

FIG. 6. (Color online) Typical TEM images of SWNTs obtained under the two gravity conditions with $p(\mathrm{He})=65 \mathrm{kPa}, I_{d} \sim 50 \mathrm{~A}$.

ments, the production location of the clusters is regarded to be the narrow region around the arc flame under both gravity conditions, where the gas temperature drops from 10000 to less than $1000 \mathrm{~K}$. Only in the narrow region around the arc flame are the carbon clusters synthesized.

SWNTs are produced using the catalyst-mixed carbon anode under the two gravity conditions with $p(\mathrm{He})=65 \mathrm{kPa}$ and $I_{d} \sim 50 \mathrm{~A}$. Figure 6 shows typical TEM images of SWNTs obtained under the two gravity conditions. There are bundles of SWNTs, dark metal catalyst particles of about $10 \mathrm{~nm}$ diameter, and an amorphous carbon layer covering the SWNTs. Figure 7 shows the number of SWNTs in the bundles of SWNTs measured from the TEM images in Fig. 6. It is confirmed that fatter bundles of SWNTs tend to be efficiently produced under the gravity-free condition. There is the possibility that the bundles of SWNTs have spherical cross sections and the back SWNTs in the bundles are not observed in these photographs. Therefore, the number in Fig. 7 shows "the number of observable SWNTs in a bundle." In order to obtain the correct number of SWNTs in each bundle, 

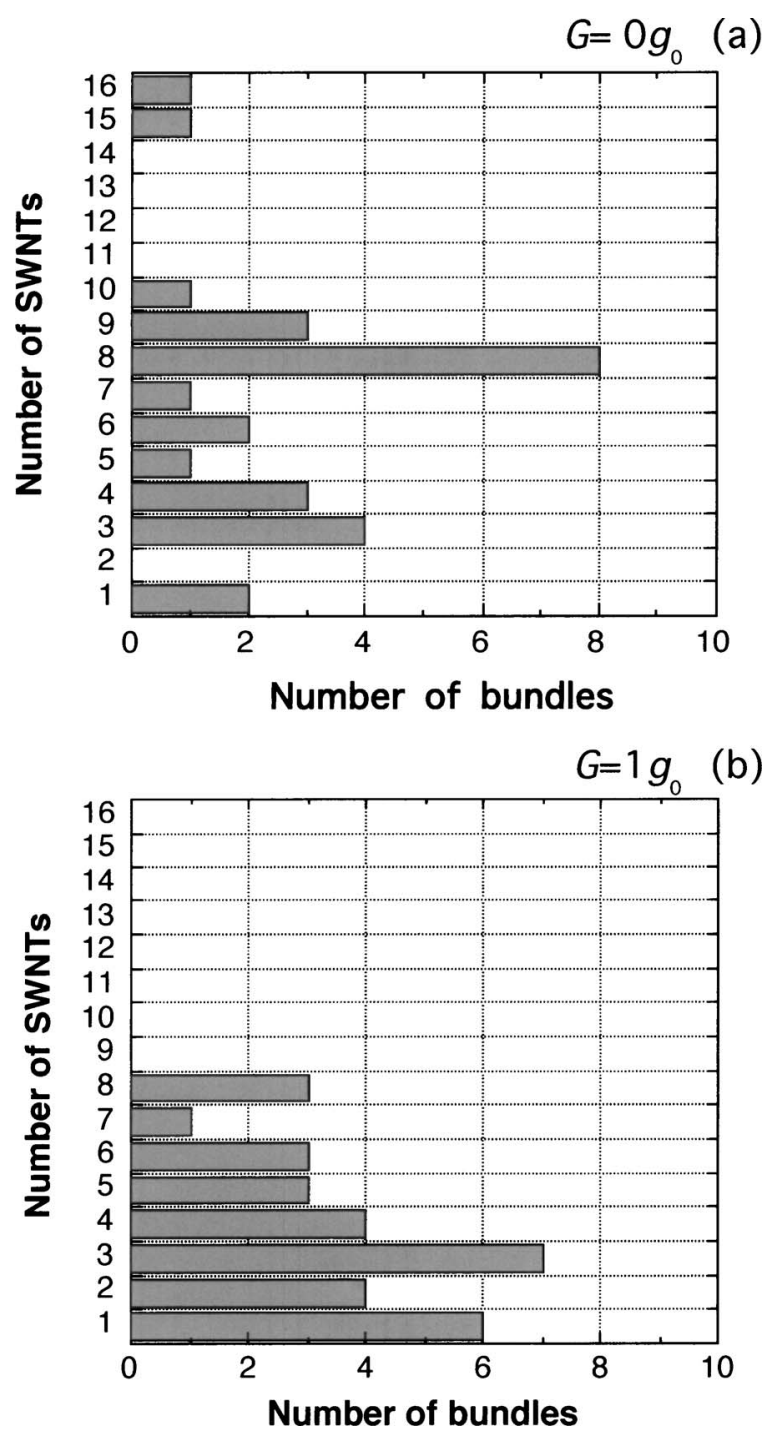

FIG. 7. (Color online) Number of observable SWNTs in a bundle counted from the TEM images in Fig. 6. The shaded SWNTs in each bundle were not counted. $p(\mathrm{He})=65 \mathrm{kPa}, I_{d} \sim 50 \mathrm{~A}$.

more precise measurement should be performed. From the measurement, it can be pointed out that the production efficiency of SWNTs is considerably increased under the gravity-free condition. SWNT diameter distributions for the two gravity conditions are measured. Under $0 g_{0}$, the mean diameter is about $1.5 \mathrm{~nm}$, while under $1 g_{0}$, it is about $1.3 \mathrm{~nm}$.

We expect the production of longer SWNTs under the gravity-free condition. However, it is difficult to measure the length distribution from the TEM images. Further study is necessary to elucidate the conditions under which longer SWNTs are produced in the gravity-free environment.

\section{SUMMARY}

The thermal properties of arc discharge are measured on board a jet plane and the expansion of the hot-gas region is clearly observed under the gravity-free condition, where lower temperature gradient and slow expansion of the hotgas region are expected. By the Mie scattering method, the production location and the diffusion properties of carbon clusters are observed. The results are consistent with the theoretical prediction. The clusters appear around the arc flame and they slowly, homogeneously, and randomly move around under the gravity-free condition. Large bundles of fatter SWNTs tend to be produced under the gravity-free condition. It is expected that under a longer and ideal gravity-free condition, clearer improvement of SWNT production will be achieved.

\section{ACKNOWLEDGMENTS}

The authors thank Diamond Air Service for the operation of the jet planes. This study was supported by the Japan Space Forum. This study is partly supported by the Nanotechnology Project of Ministry of Education, Culture, Sports, Science and Technology (MEXT), Japan.

${ }^{1}$ H. W. Kroto, J. R. Heath, S. C. O’Brien, R. F. Curl, and R. E. Smalley, Nature (London) 318, 162 (1985).

${ }^{2}$ W. Kraetschmer, L. D. Lamb, K. Fostirpoulos, and D. R. Huffman, Nature (London) 347, 354 (1990).

${ }^{3}$ S. Iijima, Nature (London) 354, 56 (1991).

${ }^{4}$ S. Iijima and T. Ichihashi, Nature (London) 363, 603 (1993).

${ }^{5}$ T. Mieno, Jpn. J. Appl. Phys., Part 2 37, L761 (1998).

${ }^{6}$ M. Kanai, A. Koshio, H. Shinohara, T. Mieno, A. Kasuya, Y. Ando, and X. Zhao, Appl. Phys. Lett. 79, 2967 (2001).

${ }^{7}$ T. Mieno, Plasma Phys. Controlled Fusion 46, 211 (2004).

${ }^{8}$ T. Mieno, Jpn. J. Appl. Phys., Part 2 35, L591 (1996).

${ }^{9}$ T. Mieno, Jpn. J. Appl. Phys., Part 2 42, L960 (2003).

${ }^{10}$ T. Mieno, T. Mizutani, and M. Takeguchi, Novel Materials Processing by Advanced Electromagnetic Energy Sources, edited by S. Miyake (Elsevier, Oxford, 2005), p. 45.

${ }^{11}$ E. L. Cussler, Diffusion: Mass Transfer in Fluid Systems (Cambridge University Press, New York, 1984), p. 19.

${ }^{12}$ R. B. Bird, W. E. Stewart, and E. N. Lightfoot, Transport Phenomena (Wiley, New York, 1960), p. 510.

${ }^{13}$ C. A. J. Fletcher, Computational Techniques for Fluid Dynamics 2 (Springer, New York, 1988), p. 337.

${ }^{14}$ R. Peyret and T. D. Taylor, Computational Methods for Fluid Flow (Springer, New York, 1983), p. 160.

${ }^{15}$ S. Usuba, H. Yokoi, and Y. Kakudate, J. Appl. Phys. 91, 10051 (2002). 